\title{
Synthesis and Pharmacology Evolution of New Coumarin Clubbed Carbamodithioate Moiety using Biocatalyst
}

\author{
KEYURKUMAR A. KAMANI and KANUPRASAD D. PATEL ${ }^{*}$ \\ V. P. \& R. P. T. P. Science College, Sardar Patel University, \\ Vallabh Vidyanagar-388 120, Gujarat, India \\ keyurkamani28@gmail.com;drkdpatel64@yahoo.co.in
}

Received 25 July 2016 / Accepted 3 September 2016

\begin{abstract}
A series of structurally new coumarin clubbed carbamodithioate derivatives 7a-t were designed and synthesized by multistep synthesis using biocatalyst Baker's Yeast. All the new compounds were characterized by mass, ${ }^{1} \mathrm{H}$ and ${ }^{13} \mathrm{C}$ NMR and elemental analysis. Furthermore, compound 7a-t evaluated for their in vitro antibacterial and antitubercular activities. The results indicated that some of the synthesized compounds posses promising antimicrobial activity against some gram-positive and gram-negative bacteria. Some compounds displayed good activity against Mycobacterium tuberculosis $H 37 R v$ as compared to standard drug.
\end{abstract}

Keywords: Baker's yeast, Carbamodithioate, Coumarin, Antibacterial activity, Antitubercular activity

\section{Introduction}

Biocatalyst has attracted the attention of organic as well as medical chemist for their selectivity and various organic transformations and baker's yeast prove itself useful in organic as well as medicinal chemistry ${ }^{1}$. Defiantly we can say that most of natural product and widely distributed pharmaceutical agents contain sulphur as heteroatom in heterocyclic structure. Carbamodithioate are such heterocyclic system which is found to have interesting chemistry and wide utility ${ }^{2}$.

Drug design and development provide useful guidance to club various active moiety to achieve active molecule which is called pharmacophore hybridisation ${ }^{3}$. On this basis we made an attempt to synthesis and pharmacology evolution of new coumarin clubbed carbamodithioate moiety using biocatalyst. Coumarin and carbamodithioate are clubbed in a way to get desired molecule to obtain best result and it is mentioned here.

\section{Experimental}

All the melting points were taken in scientific melting point apparatus and was uncorrected. Silica gel-G coated aluminums plates (Merck) were used to check purity and completion of 
the reaction and spots were visualized by exposing the dry plates in iodine vapours as well as UV light used for it. Mass spectra of intermediates and final products were scanned on a Shimadzu LCMS 2010 spectrometer. 1H \& 13C NMR spectra on a Bruker's WM 400 FT MHz NMR instrument using DMSO-d6 as solvent and TMS as internal reference (chemical shifts in $\delta \mathrm{ppm})$. The elemental analysis $(\mathrm{C}, \mathrm{H}$ and $\mathrm{N}$ ) of compounds was performed on Carlo Erba-1108 elemental analyzer.

\section{General procedure of synthesis of carbamodithioate derivatives (7a-t)}

In the round bottom flask, a mixture of 5a-t $(0.069 \mathrm{~mol})$ and $6(0.069 \mathrm{~mol})$ was dissolved into the ethanol $(25 \mathrm{~mL})$ and allowed to reflux for $6-8 \mathrm{~h}$. Solid product obtained as precipitate in reaction mass was checked by TLC and confirmed the formation of compound 7a-t. In work up process, excess ethanol was removed by distillation, solid residue was treated with cold water, filtered, dried and purification was achieved by crystallization in ethanol to obtain pure compound 7a-t (Table 1). Detailed characterisation of series 7a-t given below

Table 1. Physical data and information of substitution of 7a-t

\begin{tabular}{cccccc}
\hline S. No. & Compound & $\mathrm{R}$ & $\mathrm{R}_{1}$ & \% Yield & $\mathrm{Mp},{ }^{\circ} \mathrm{C}$ \\
\hline 1 & $\mathbf{7 a}$ & $\mathrm{H}$ & $\mathrm{H}$ & 75 & 155 \\
2 & $\mathbf{7 b}$ & $\mathrm{H}$ & $\mathrm{NO}_{2}$ & 80 & 142 \\
3 & $\mathbf{7 c}$ & $\mathrm{H}$ & $\mathrm{Cl}$ & 80 & 140 \\
4 & $\mathbf{7 d}$ & $\mathrm{H}$ & $\mathrm{F}$ & 85 & 132 \\
5 & $\mathbf{7 e}$ & $\mathrm{Cl}$ & $\mathrm{H}$ & 70 & 158 \\
6 & $\mathbf{7 f}$ & $\mathrm{Cl}$ & $\mathrm{NO}_{2}$ & 95 & 168 \\
7 & $\mathbf{7 g}$ & $\mathrm{Cl}$ & $\mathrm{Cl}$ & 75 & 170 \\
8 & $\mathbf{7 h}$ & $\mathrm{Cl}$ & $\mathrm{F}$ & 78 & 121 \\
9 & $\mathbf{7 i}$ & $\mathrm{Br}$ & $\mathrm{H}$ & 84 & 135 \\
10 & $\mathbf{7 j}$ & $\mathrm{Br}$ & $\mathrm{NO}_{2}$ & 80 & 140 \\
11 & $\mathbf{7 k}$ & $\mathrm{Br}$ & $\mathrm{Cl}$ & 90 & 169 \\
12 & $\mathbf{7 l}$ & $\mathrm{Br}$ & $\mathrm{F}$ & 92 & 147 \\
13 & $\mathbf{7 m}$ & $\mathrm{F}$ & $\mathrm{H}$ & 89 & 158 \\
14 & $\mathbf{7 n}$ & $\mathrm{F}$ & $\mathrm{NO}_{2}$ & 78 & 135 \\
15 & $\mathbf{7 o}$ & $\mathrm{F}$ & $\mathrm{Cl}$ & 76 & 159 \\
16 & $\mathbf{7 p}$ & $\mathrm{F}$ & $\mathrm{F}$ & 90 & 148 \\
17 & $\mathbf{7 q}$ & $\mathrm{Me}$ & $\mathrm{H}$ & 92 & 151 \\
18 & $\mathbf{7 r}$ & $\mathrm{Me}$ & $\mathrm{NO}_{2}$ & 90 & 130 \\
19 & $\mathbf{7 s}$ & $\mathrm{Me}$ & $\mathrm{Cl}$ & 95 & 124 \\
20 & $\mathbf{7 t}$ & $\mathrm{Me}$ & $\mathrm{F}$ & 60 & 145 \\
\hline
\end{tabular}

2-Oxo-2-(2-oxo-2H-chromen-3-yl)ethyl benzyl(phenyl)carbamodithioate (7a)

Chemical Formula: $\mathrm{C}_{25} \mathrm{H}_{19} \mathrm{NO}_{3} \mathrm{~S}_{2} \mathrm{MS}(\mathrm{m} / \mathrm{z}): 445.51\left[\mathrm{M}^{+}\right] .{ }^{1} \mathrm{H}$ NMR (400 MHz, DMSO) $\delta$ $8.32(\mathrm{~m}, 1 \mathrm{H}), 7.49(\mathrm{~m}, 1 \mathrm{H}), 7.51-6.56(\mathrm{~m}, 13 \mathrm{H}), 4.91(\mathrm{~s}, 2 \mathrm{H}), 4.20(\mathrm{~s}, 2 \mathrm{H}) .{ }^{13} \mathrm{C}$ NMR $\delta$ $193.03,160.25,154.11,142.22,137.12,132.72,129.45,128.24,128.67,128.12,126.50$, 125.05, 120.51, 118.23, 117.87, 55.86, 42.53. Elemental Analysis: calculated C, 67.39; H, $4.30 ; \mathrm{N}, 3.14 \%$ found $\mathrm{C}, 67.34 ; \mathrm{H}, 4.37 ; \mathrm{N}, 3.06 \%$.

\section{2-Oxo-2-(2-oxo-2H-chromen-3-yl)ethyl (4-nitrobenzyl)(phenyl)carbamodithioate(7b)}

Chemical Formula: $\mathrm{C}_{25} \mathrm{H}_{18} \mathrm{~N}_{2} \mathrm{O}_{5} \mathrm{~S}_{2} \mathrm{MS}(\mathrm{m} / \mathrm{z}): 490.52\left[\mathrm{M}^{+}\right] .{ }^{1} \mathrm{H}$ NMR (400 MHz, DMSO) $\delta$ 8.22-6.98 (m, 14H), 5.15 (s, 2H), $4.46(\mathrm{~s}, 2 \mathrm{H}) .{ }^{13} \mathrm{C}$ NMR $\delta$ 193.06, 160.64, 154.24, 147.26, 
$143.64,142.28,132.69,129.25,128.55,127.63,126.55,125.12,123.59,120.54,118.12$, 117.47, 53.75, 41.52. Elemental Analysis: calculated C, 61.21; H, 3.70; N, 5.71\% found C, $61.12 ; \mathrm{H}, 3.78 ; \mathrm{N}, 5.82 \%$.

2-Oxo-2-(2-oxo-2H-chromen-3-yl)ethyl (4-chlorobenzyl)(phenyl) carbamodithioate(7c)

Chemical Formula: $\mathrm{C}_{25} \mathrm{H}_{18} \mathrm{ClNO}_{3} \mathrm{~S}_{2} \mathrm{MS}(\mathrm{m} / \mathrm{z}): 479.94\left[\mathrm{M}^{+}\right] .{ }^{1} \mathrm{H}$ NMR (400 MHz, DMSO) $\delta$ 8.19-7.05 (m, 14H), 5.21 (s, 2H), $4.43(\mathrm{~s}, 2 \mathrm{H}) .{ }^{13} \mathrm{C}$ NMR $\delta 193.25,160.54,154.54,142.27$, 135.06, 132.64, 130.54, 129.45, 128.84, 127.15, 126.21, 125.18, 120.52, 118.15, 117.25, 53.26, 41.92. Elemental Analysis: calculated C, 62.56; H, 3.78; N, 2.92\% found C, 62.49; H, $3.86 ; \mathrm{N}, 2.99 \%$.

2-Oxo-2-(2-oxo-2H-chromen-3-yl)ethyl (4-fluorobenzyl)(phenyl) carbamodithioate(7d) Chemical Formula: $\mathrm{C}_{25} \mathrm{H}_{18} \mathrm{FNO}_{3} \mathrm{~S}_{2} \mathrm{MS}(\mathrm{m} / \mathrm{z}): 463.57\left[\mathrm{M}^{+}\right] .{ }^{1} \mathrm{H}$ NMR $(400 \mathrm{MHz}, \mathrm{DMSO}) \delta$ 8.21-6.92 (m, 14H), 5.08 (s, 2H), 4.36 (s, 2H). ${ }^{13} \mathrm{C}$ NMR $\delta$ 194.01, 164.26, 161.64, 160.94, $154.13,142.17,132.87,131.50,130.61,129.56,127.85,126.54,125.18,120.55,118.68$, 117.19, 115.74, 114.47, 53.83, 42.57 Elemental Analysis: calculated C, 64.78; H, 3.91; N, $3.02 \%$ found $\mathrm{C}, 64.83 ; \mathrm{H}, 3.84 ; \mathrm{N}, 3.13 \%$.

2-Oxo-2-(2-oxo-2H-chromen-3-yl)ethyl benzyl(4-chlorophenyl)carbamodithioate(7e)

Chemical Formula: $\mathrm{C}_{25} \mathrm{H}_{18} \mathrm{ClNO}_{3} \mathrm{~S}_{2} \mathrm{MS}(\mathrm{m} / \mathrm{z})$ : $479.93\left[\mathrm{M}^{+}\right] .{ }^{1} \mathrm{H}$ NMR (400 MHz, DMSO) $\delta$ 8.29-6.96 (m, 14H), 5.20 (s, 2H), 4.32 (s, 2H). ${ }^{13} \mathrm{C}$ NMR $\delta$ 193.26, 160.71, 154.97, 140.67, $137.84,132.57,132.28,129.62,128.69,128.22$, 127.34, 127.17, 125.21, 120.26, 118.57, 117.58, 52.68, 42.59. Elemental Analysis: calculated C, 62.56; H, 3.78; N, 2.92\% found C, $62.67 ; \mathrm{H}, 3.84 ; \mathrm{N}, 2.87 \%$.

2-Oxo-2-(2-oxo-2H-chromen-3-yl)ethyl (4-chlorophenyl)(4-nitrobenzyl) carbamodithioate $(7 \boldsymbol{f})$

Chemical Formula: $\mathrm{C}_{25} \mathrm{H}_{17} \mathrm{ClN}_{2} \mathrm{O}_{5} \mathrm{~S}_{2} \mathrm{MS}(\mathrm{m} / \mathrm{z}): 524.95\left[\mathrm{M}^{+}\right] .{ }^{1} \mathrm{H}$ NMR (400 MHz, DMSO) $\delta$ 8.37-6.99 (m, 13H), 5.02 (s, 2H), $4.33(\mathrm{~s}, 2 \mathrm{H}) .{ }^{13} \mathrm{C}$ NMR $\delta 193.80,160.69,154.22,147.50$, $143.99,140.82,132.64,132.38,129.57,128.86,128.38,127.65,125.24,123.73,120.58$, 118.85, 117.12, 53.87, 41.57. Elemental Analysis: calculated C, 57.20; H, 3.26; N, 5.34\% found C, 57.29; H, 3.18; N, 5.42\%.

\section{2-Oxo-2-(2-oxo-2H-chromen-3-yl)ethyl (4-chlorobenzyl)(4-chlorophenyl)} carbamodithioate $(\mathbf{7 g})$

Chemical Formula: $\mathrm{C}_{25} \mathrm{H}_{17} \mathrm{Cl}_{2} \mathrm{NO}_{3} \mathrm{~S}_{2} \mathrm{MS}(\mathrm{m} / \mathrm{z}): 514.41\left[\mathrm{M}^{+}\right] .{ }^{1} \mathrm{H}$ NMR (400 MHz, DMSO) $\delta$ 8.34-6.04 (m, 13H), $5.18(\mathrm{~s}, 2 \mathrm{H}), 4.28(\mathrm{~s}, 2 \mathrm{H}) .{ }^{13} \mathrm{C}$ NMR $\delta 193.66,160.82,154.36,140.67$, $135.18,133.08,132.39,130.27,129.52,128.96,128.23,127.17,125.15,120.26,118.89$, 117.25, 56.12, 42.81. Elemental Analysis: calculated C, 58.37; H, 3.33; N, 2.72\% found C, $58.28 ; \mathrm{H}, 3.41 ; \mathrm{N}, 2.62 \%$.

\section{2-Oxo-2-(2-oxo-2H-chromen-3-yl)ethyl (4-chlorophenyl)(4-fluorobenzyl) carbamodithioate( $7 \boldsymbol{h})$}

Chemical Formula: $\mathrm{C}_{25} \mathrm{H}_{17} \mathrm{ClFNO}_{3} \mathrm{~S}_{2} \mathrm{MS}(\mathrm{m} / \mathrm{z})$ : $497.92\left[\mathrm{M}^{+}\right] .{ }^{1} \mathrm{H}$ NMR (400 MHz, DMSO) $\delta$ 8.18-6.91 (m, 13H), 5.07 (s, 2H), $4.41(\mathrm{~s}, 2 \mathrm{H}) .{ }^{13} \mathrm{C}$ NMR $\delta 193.61,164.78,161.22,160.64$, $154.15,140.24,133.17,132.89,131.59,130.68,129.43,128.29,127.12,125.39,120.73$, 118.38, 117.35, 115.19, 114.65, 55.64, 42.34. Elemental Analysis: calculated C, 60.30; H, $3.44 ; \mathrm{N}, 2.81 \%$ found $\mathrm{C}, 60.41 ; \mathrm{H}, 3.39 ; \mathrm{N}, 2.87 \%$. 


\section{2-Oxo-2-(2-oxo-2H-chromen-3-yl)ethyl benzyl(4-bromophenyl)carbamodithioate(7i)}

Chemical Formula: $\mathrm{C}_{25} \mathrm{H}_{18} \mathrm{BrNO}_{3} \mathrm{~S}_{2} \mathrm{MS}(\mathrm{m} / \mathrm{z}): 524.48\left[\mathrm{M}^{+}\right] .{ }^{1} \mathrm{H}$ NMR (400 MHz, DMSO) $\delta$ 8.19-6.99 (m, 14H), 5.05 (s, 2H), 4.27 (s, 2H). ${ }^{13} \mathrm{C}$ NMR $\delta 193.23,160.56,154.38,141.33$, $137.34,132.74,131.13,129.64,128.98,128.86,128.14,126.78,125.15,120.38,118.98$, 118.26, 117.68, 54.57, 43.69. Elemental Analysis: calculated C, 57.26; H, 3.46; N, 2.67\% found $\mathrm{C}, 57.32 ; \mathrm{H}, 3.33 ; \mathrm{N}, 2.74 \%$.

\section{2-Oxo-2-(2-oxo-2H-chromen-3-yl)ethyl (4-bromophenyl)(4-nitrobenzyl) carbamodithioate $(\mathbf{j} \mathbf{j})$}

Chemical Formula: $\mathrm{C}_{25} \mathrm{H}_{17} \mathrm{BrN}_{2} \mathrm{O}_{5} \mathrm{~S}_{2} \mathrm{MS}(\mathrm{m} / \mathrm{z}): 569.44\left[\mathrm{M}^{+}\right] .{ }^{1} \mathrm{H}$ NMR (400 MHz, DMSO) $\delta$ 8.34-7.04 (m, 13H), $5.01(\mathrm{~s}, 2 \mathrm{H}), 4.31(\mathrm{~s}, 2 \mathrm{H}) .{ }^{13} \mathrm{C}$ NMR $\delta 193.32,160.62,154.26,147.26$, $143.64,141.16,132.72,131.22,129.43,128.87,128.16,126.58,125.27,123.75,120.78$, 118.66, 118.11, 117.34, 55.17, 44.14. Elemental Analysis: calculated C, 52.73; H, 3.01; N, $4.92 \%$ found $\mathrm{C}, 52.62 ; \mathrm{H}, 3.13 ; \mathrm{N}, 4.81 \%$.

\section{2-Oxo-2-(2-oxo-2H-chromen-3-yl)ethyl (4-bromophenyl)(4-chlorobenzyl) carbamodithioate( $7 \boldsymbol{k})$}

Chemical Formula: $\mathrm{C}_{25} \mathrm{H}_{17} \mathrm{BrClNO}_{3} \mathrm{~S}_{2} \mathrm{MS}(\mathrm{m} / \mathrm{z}): 558.84\left[\mathrm{M}^{+}\right] .{ }^{1} \mathrm{H}$ NMR (400 MHz, DMSO) $\delta 8.35-6.97(\mathrm{~m}, 13 \mathrm{H}), 5.11(\mathrm{~s}, 2 \mathrm{H}), 4.38(\mathrm{~s}, 2 \mathrm{H}) .{ }^{13} \mathrm{C}$ NMR $\delta 193.66,160.70,154.31,141.26$, $135.17,132.92,131.28,130.15,129.52,128.89,128.12,126.77,125.18,120.58,118.63$, 118.13, 117.36, 55.69, 41.35. Elemental Analysis: calculated C, 53.73; H, 3.07; N, $2.51 \%$ found C, 53.68; H, 3.16; N, 2.43\%.

\section{2-Oxo-2-(2-oxo-2H-chromen-3-yl)ethyl (4-bromophenyl)(4-fluorobenzyl) carbamodithioate $(7 \mathrm{l})$}

Chemical Formula: $\mathrm{C}_{25} \mathrm{H}_{17} \mathrm{BrFNO}_{3} \mathrm{~S}_{2} \mathrm{MS}(\mathrm{m} / \mathrm{z}): 542.48\left[\mathrm{M}^{+}\right] .{ }^{1} \mathrm{H}$ NMR (400 MHz, DMSO) $\delta 8.20-6.91(\mathrm{~m}, 13 \mathrm{H}), 5.18(\mathrm{~s}, 2 \mathrm{H}), 4.39(\mathrm{~s}, 2 \mathrm{H}) .{ }^{13} \mathrm{C}$ NMR $\delta 193.34,164.68,161.74,160.50$, $154.36,141.16,132.83,131.58,131.36,130.78,129.25,128.12,126.66,125.35,120.66$, 118.40, 118.26, 117.12, 115.54, 114.38, 56.71, 43.37. Elemental Analysis: calculated C, $55.36 ; \mathrm{H}, 3.16 ; \mathrm{N}, 2.58 \%$ found $\mathrm{C}, 55.28 ; \mathrm{H}, 3.22 ; \mathrm{N}, 2.42 \%$.

\section{2-Oxo-2-(2-oxo-2H-chromen-3-yl)ethyl benzyl(4-fluorophenyl)carbamodithioate(7m)}

Chemical Formula: $\mathrm{C}_{25} \mathrm{H}_{18} \mathrm{FNO}_{3} \mathrm{~S}_{2} \mathrm{MS}(\mathrm{m} / \mathrm{z}): 463.57\left[\mathrm{M}^{+}\right] .{ }^{1} \mathrm{H}$ NMR $(400 \mathrm{MHz}, \mathrm{DMSO}) \delta$ 8.31-6.96 (m, 14H), 5.01 (s, 2H), 4.37 (s, 2H). ${ }^{13} \mathrm{C}$ NMR $\delta$ 193.91, 164.48, 161.74, 160.22, $154.25,139.50,137.57,132.64,129.65,128.96,128.75,128.15,127.54,125.21,120.57$, 118.11, 117.58, 116.66, 115.50, 54.84, 42.55. Elemental Analysis: calculated C, 64.78; H, $3.91 ; \mathrm{N}, 3.02 \%$ found $\mathrm{C}, 64.84 ; \mathrm{H}, 3.82 ; \mathrm{N}, 3.11 \%$.

\section{2-Oxo-2-(2-oxo-2H-chromen-3-yl)ethyl (4-fluorophenyl)(4-nitrobenzyl) carbamodithioate $(7 \boldsymbol{n})$}

Chemical Formula: $\mathrm{C}_{25} \mathrm{H}_{17} \mathrm{FN}_{2} \mathrm{O}_{5} \mathrm{~S}_{2} \mathrm{MS}(\mathrm{m} / \mathrm{z}): 508.56\left[\mathrm{M}^{+}\right] .{ }^{1} \mathrm{H}$ NMR (400 MHz, DMSO) $\delta$ 8.14-6.95 (m, 13H), 5.13 (s, 2H), $4.40(\mathrm{~s}, 2 \mathrm{H}) .{ }^{13} \mathrm{C}$ NMR $\delta 193.67,164.43,161.92,160.67$, $154.27,147.24,143.49,139.71,132.57,129.69,128.87,128.15,127.68,125.12,123.63$, 120.57, 118.52, 117.47, 116.24, 115.54, 53.38, 43.57. Elemental Analysis: calculated C, $59.05 ; \mathrm{H}, 3.37 ; \mathrm{N}, 5.51 \%$ found $\mathrm{C}, 59.16 ; \mathrm{H}, 3.46 ; \mathrm{N}, 5.42 \%$. 


\section{2-Oxo-2-(2-oxo-2H-chromen-3-yl)ethyl (4-chlorobenzyl)(4-fluorophenyl) carbamodithioate(7o)}

Chemical Formula: $\mathrm{C}_{25} \mathrm{H}_{17} \mathrm{ClFNO}_{3} \mathrm{~S}_{2} \mathrm{MS}(\mathrm{m} / \mathrm{z})$ : $497.94\left[\mathrm{M}^{+}\right] .{ }^{1} \mathrm{H}$ NMR (400 MHz, DMSO) $\delta$ 8.08-6.97 (m, 13H), 5.06 (s, 2H), 4.39 (s, 2H). ${ }^{13} \mathrm{C}$ NMR $\delta 193.86,164.47,161.82,160.66$, $154.44,139.52$, 135.17, 132.43, 130.65, 129.41, 128.64, 128.18, 127.68, 125.20, 120.71, 118.54, 117.48, 116.25, 115.87, 55.68, 41.64. Elemental Analysis: calculated C, 60.30; H, $3.44 ; \mathrm{N}, 2.81 \%$ found $\mathrm{C}, 60.28 ; \mathrm{H}, 3.57 ; \mathrm{N}, 2.92 \%$.

\section{2-Oxo-2-(2-oxo-2H-chromen-3-yl)ethyl (4-fluorobenzyl)(4-fluorophenyl) carbamodithioate $(7 \boldsymbol{p})$}

Chemical Formula: $\mathrm{C}_{25} \mathrm{H}_{17} \mathrm{~F}_{2} \mathrm{NO}_{3} \mathrm{~S}_{2} \mathrm{MS}(\mathrm{m} / \mathrm{z})$ : $481.53\left[\mathrm{M}^{+}\right] .{ }^{1} \mathrm{H}$ NMR (400 MHz, DMSO) $\delta$ 8.31-6.93 (m, 13H), 5.09 (s, 2H), 4.37 (s, 2H). ${ }^{13} \mathrm{C}$ NMR $\delta 193.68,164.87,164.36,161.93$, $161.52,160.86,154.15,139.59,132.90,131.41,130.82,129.59,128.19,127.64,125.15$, $120.83,118.66,117.27,116.51,115.98,115.65,114.64,53.87,42.22$. Elemental Analysis: calculated C, 62.36; H, 3.56; N, 2.91\% found C, 62.41; H, 3.48; N, 2.97\%.

\section{2-Oxo-2-(2-oxo-2H-chromen-3-yl)ethyl benzyl(p-tolyl)carbamodithioate(7q)}

Chemical Formula: $\mathrm{C}_{26} \mathrm{H}_{21} \mathrm{NO}_{3} \mathrm{~S}_{2} \mathrm{MS}(\mathrm{m} / \mathrm{z}): 459.54\left[\mathrm{M}^{+}\right] .{ }^{1} \mathrm{H}$ NMR (400 MHz, DMSO) $\delta$ 8.19-6.90 (m, 14H), 5.16 (s, 2H), 4.32 (s, 2H) $2.24(\mathrm{~s}, 3 \mathrm{H}) .{ }^{13} \mathrm{C}$ NMR $\delta 193.78,160.85$, $154.23,140.53,137.24,136.56,132.75,129.45,128.96,128.72,128.12,125.18,122.52$, 120.69, 118.22, 117.12, 55.67, 42.53, 23.15. Elemental Analysis: calculated C, 67.95; H, $4.61 ; \mathrm{N}, 3.05 \%$ found $\mathrm{C}, 67.83 ; \mathrm{H}, 4.55 ; \mathrm{N}, 3.19 \%$.

\section{2-Oxo-2-(2-oxo-2H-chromen-3-yl)ethyl (4-nitrobenzyl)(p-tolyl)carbamodithioate(7r)}

Chemical Formula: $\mathrm{C}_{26} \mathrm{H}_{20} \mathrm{~N}_{2} \mathrm{O}_{5} \mathrm{~S}_{2} \mathrm{MS}(\mathrm{m} / \mathrm{z}): 504.53\left[\mathrm{M}^{+}\right] .{ }^{1} \mathrm{H}$ NMR (400 MHz, DMSO) $\delta$ 8.21-6.93 (m, 13H), $5.18(\mathrm{~s}, 2 \mathrm{H}), 4.37(\mathrm{~s}, 2 \mathrm{H}) 2.29(\mathrm{~s}, 3 \mathrm{H}) .{ }^{13} \mathrm{C}$ NMR $\delta$ 193.89, 160.67, $154.50,147.34,143.52,140.49,136.68,132.82,129.78,128.62,128.17,125.24,123.87$, $122.58,120.70,118.23,117.19,55.67,42.58,22.92$. Elemental Analysis: calculated C, $61.89 ; \mathrm{H}, 4.00 ; \mathrm{N}, 5.55 \%$ found $\mathrm{C}, 61.96 ; \mathrm{H}, 4.12 ; \mathrm{N}, 5.42 \%$.

\section{2-Oxo-2-(2-oxo-2H-chromen-3-yl)ethyl (4-chlorobenzyl)(p-tolyl)carbamodithioate(7s)}

Chemical Formula: $\mathrm{C}_{26} \mathrm{H}_{20} \mathrm{ClNO}_{3} \mathrm{~S}_{2} \mathrm{MS}(\mathrm{m} / \mathrm{z})$ : $494.05\left[\mathrm{M}^{+}\right] .{ }^{1} \mathrm{H}$ NMR (400 MHz, DMSO) $\delta$ 8.22-6.98 (m, 13H), $5.20(\mathrm{~s}, 2 \mathrm{H}), 4.36(\mathrm{~s}, 2 \mathrm{H}) 2.26(\mathrm{~s}, 3 \mathrm{H}) .{ }^{13} \mathrm{C}$ NMR $\delta 193.84,160.87$, $154.25,140.57,136.67,135.16,132.90,130.32,129.74,128.91,128.18,125.14,122.58$, 120.25, 118.12, 117.23, 55.78, 42.46, 22.92. Elemental Analysis: calculated C, 63.21; H, $4.08 ; \mathrm{N}, 2.84 \%$ found $\mathrm{C}, 63.32 ; \mathrm{H}, 4.01 ; \mathrm{N}, 2.92 \%$.

\section{2-Oxo-2-(2-oxo-2H-chromen-3-yl)ethyl (4-fluorobenzyl)(p-tolyl)carbamodithioate(7t)}

Chemical Formula: $\mathrm{C}_{26} \mathrm{H}_{20} \mathrm{FNO}_{3} \mathrm{~S}_{2} \mathrm{MS}(\mathrm{m} / \mathrm{z}): 477.52\left[\mathrm{M}^{+}\right] .{ }^{1} \mathrm{H}$ NMR (400 MHz, DMSO) $\delta$ 8.28-6.93 (m, 13H), 5.17 (s, 2H), 4.35 (s, 2H) 2.23 (s, 3H). ${ }^{13} \mathrm{C}$ NMR $\delta$ 193.94, 164.37, $161.53,160.84,154.17,140.53,136.40,132.82,131.69,130.45,129.68,128.24,125.18$, 122.84, 120.76, 118.23, 117.27, 115.20, 114.71, 53.64, 42.52, 22.24. Elemental Analysis: calculated C, 65.39; H, 4.22; N, 2.93\% found $\mathrm{C}, 65.25 ; \mathrm{H}, 4.36 ; \mathrm{N}, 2.83 \%$.

\section{Results and Discussion}

Using substituted aldehyde (1a-d) and substituted amine (2a-e) we prepared Schiff base (3a-t) with help of baker's yeast at room temperature ${ }^{4-5}$. Here we eliminate the conventional method of 
synthesis of Schiff base and getting good result in $1^{\text {st }}$ step. In $2^{\text {nd }}$ step, reduction of Schiff base carried $^{6}$ out using sodium borohydride in methanol to obtained 4a-t. Carbon disulfide and sodium hydroxide used for synthesis of carbodithiate derivatives (5a-t $)^{7}$. Finally, active moiety 3(bromoacetyl)-2H-chromen-2-one clubbed with 5a-t using ethanol as a solvent to get desired product 7a-t in good yield ${ }^{2,8}$. Detail experimental process is given below (Scheme 1).
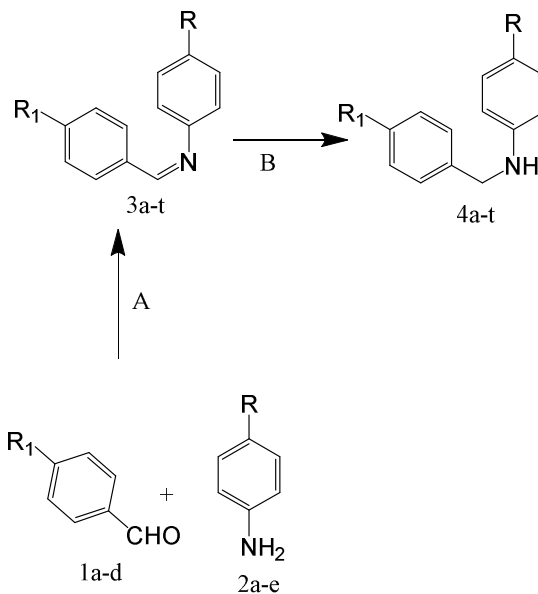

Here, $\mathrm{R}=-\mathrm{H},-\mathrm{Cl},-\mathrm{Br},-\mathrm{F},-\mathrm{Me}$

$\mathrm{R}_{1}=-\mathrm{H},-\mathrm{NO}_{2},-\mathrm{Cl},-\mathrm{F}$<smiles>[R]c1ccc(CN(C(=S)[Sn])c2ccc([R])cc2)cc1</smiles>

$\mathrm{D}$<smiles>[R]c1ccc(CN(C([AlH2])=S)c2ccc([R])cc2)cc1</smiles><smiles>COc1ccccc1C=C(C(=O)O)C(=O)CBr</smiles>

$$
\mathrm{A}=\text { ethanol, } \mathrm{AcOH}, \mathrm{B}=\mathrm{NaBH}_{4}, \mathrm{H}_{2} \mathrm{O} \text { reflux, } \mathrm{C}=\mathrm{CS}_{2} \text {, aq. } \mathrm{NaOH}, 0-5{ }^{\circ} \mathrm{C}, \mathrm{D}=6 \text {, ethanol, reflux, } 6 \text { hours }
$$

\section{Antibacterial activity}

\section{Scheme 1}

The MICs of synthesized compounds were carried out by broth micro dilution method as described by Rattan ${ }^{9}$. Antibacterial activity (Table 2) was screened against gram positive and gram negative bacterial strain ${ }^{10-13}$.

Data of antibacterial activity shows that all compound $\mathbf{7 a - t}$ are good to moderate active against gram positive and gram negative bacterial strains. More compounds are active against gram positive strains. Chemically we can classify that flouro derivatives are potent to inhibit growth of bacterial strain i.e. compound $\mathbf{7 d}, \mathbf{7 m}, \mathbf{7 o}$ and $\mathbf{7 p}$ shows highest activity. Halogenated compound $\mathbf{7} \mathbf{c}$ and $\mathbf{7 k}$ are good while less substituted compound of the series not as effective as flouro derivatives. So we carried out further antitubercular test for only active and fluorinated moiety.

\section{Antitubercular activity}

In vitro antituberculosis activity of all the newly synthesized compounds against Mycobacterium tuberculosis H37Rv strain was determined by using Lowenstein-Jensen medium (conventional method) as described by Rattan ${ }^{9}$ and the observed MIC of compounds are presented in Table 3.

Good results of antibacterial activity encourage us to go for Antitubercular activity and data are listed below. As per results we can say that only single fluorinated moiety is more active than di-flouro compound and rest of compound are moderate against M.Tuberculosis $H 37 R V$. Thus, compound $\mathbf{7 d}$ and $\mathbf{7} \mathbf{m}$ are best compound of series. 
Table 2. Antibacterial Activity (Minimal Inhibition Concentration, MIC, $\mu \mathrm{g} / \mathrm{mL}$ )

\begin{tabular}{cccccccc}
\hline S. No. & $\begin{array}{c}\text { E.c. } \\
(-v e)\end{array}$ & $\begin{array}{c}\text { P.a } \\
(-v e)\end{array}$ & $\begin{array}{c}\text { Kl.pn. } \\
(-v e)\end{array}$ & $\begin{array}{c}\text { S.ty } \\
(-v e)\end{array}$ & $\begin{array}{c}\text { S.a. } \\
(+v e)\end{array}$ & $\begin{array}{c}\text { S.py. } \\
(+v e)\end{array}$ & $\begin{array}{c}\text { B.s. } \\
(+v e)\end{array}$ \\
\hline $\mathbf{7 a}$ & 500 & 500 & 500 & 500 & 500 & 500 & 500 \\
$\mathbf{7 b}$ & 500 & 200 & 500 & 500 & 500 & 500 & 250 \\
$\mathbf{7 c}$ & 100 & 500 & 500 & 250 & 62.5 & 62.5 & 200 \\
$\mathbf{7 d}$ & 500 & 200 & 500 & 500 & 500 & 500 & 250 \\
$\mathbf{7 e}$ & 500 & 500 & 500 & 500 & 500 & 500 & 500 \\
$\mathbf{7 f}$ & 500 & 500 & 500 & 500 & 500 & 500 & 500 \\
$\mathbf{7 g}$ & 500 & 500 & 500 & 500 & 500 & 500 & 500 \\
$\mathbf{7 h}$ & 250 & 200 & 100 & 62.5 & 200 & 200 & 250 \\
$\mathbf{7 i}$ & 500 & 500 & 500 & 500 & 500 & 500 & 500 \\
$\mathbf{7 j}$ & 500 & 500 & 500 & 500 & 500 & 500 & 500 \\
$\mathbf{7 k}$ & 250 & 200 & 200 & 250 & 100 & 200 & 100 \\
$\mathbf{7 l}$ & 500 & 500 & 500 & 500 & 500 & 500 & 500 \\
$\mathbf{7 m}$ & 200 & 200 & 250 & 250 & 200 & 200 & 200 \\
$\mathbf{7 n}$ & 500 & 500 & 500 & 500 & 500 & 500 & 500 \\
$\mathbf{7 0}$ & 100 & 200 & 200 & 250 & 100 & 100 & 100 \\
$\mathbf{7 p}$ & 62.5 & 100 & 62.5 & 62.5 & 100 & 100 & 62.5 \\
$\mathbf{7 q}$ & 500 & 500 & 500 & 500 & 200 & 500 & 500 \\
$\mathbf{7 r}$ & 250 & 500 & 500 & 500 & 500 & 250 & 500 \\
$\mathbf{7 s}$ & 500 & 250 & 250 & 500 & 500 & 500 & 500 \\
$\mathbf{7 t}$ & 500 & 500 & 250 & 500 & 500 & 250 & 500 \\
Gentamycin & 0.05 & 1 & 0.05 & 1 & 0.25 & 0.5 & 0.5 \\
Ampicillin & 100 & 100 & 100 & 100 & 250 & 100 & 100 \\
Chloramphenicol & 50 & 50 & 50 & 50 & 50 & 50 & 50 \\
Ciprofloxacin & 25 & 25 & 25 & 25 & 50 & 50 & 50 \\
Norfloxacin & 10 & 10 & 10 & 10 & 10 & 10 & 10 \\
\hline
\end{tabular}

E.c. $=$ E. Coli(MTCC-443); P.a. $=$ P. Aeruginosa $(M T C C-1688) ;$ Kl.pn. $=$ Kl. Pneumoniae $(M T C C-109)$; S.ty. $=$ S. Typhi(MTCC-98); S.a. $=$ S. Aureus(MTCC-96); S.py. $=$ S. Pyogenus (MTCC-442); B.s.=B. Subtilis(MTCC-441)

Table 3. Antitubercular Activity (Minimal Inhibition Concentration, MIC, $\mu \mathrm{g} / \mathrm{mL}$ )

\begin{tabular}{ccc}
\hline S. No & M. tuberculosis H37RV & \% Inhibition \\
\hline $\mathbf{7 c}$ & 500 & 98 \\
$\mathbf{7 d}$ & 62.5 & 98 \\
$\mathbf{7 h}$ & 500 & 98 \\
$\mathbf{7 i}$ & 500 & 98 \\
$\mathbf{7 k}$ & 1000 & 98 \\
$\mathbf{7 m}$ & 50 & 99 \\
$\mathbf{7 0}$ & 250 & 98 \\
$\mathbf{7 p}$ & 250 & 99 \\
Isoniazid & 40 & 98 \\
Rifampicin & 0.20 & 99 \\
\hline
\end{tabular}

M. tuberculosis H37RV (MTCC-200)

\section{Conclusion}

In present study shows benefits of biocatalyst as well as new approach for synthesis can eliminate traditional and tedious synthesis protocol. Microbial study of presented 
compounds is very interesting and flouro derivative are hit molecules among the series and shows good antibacterial and antitubercular activity. Further investigation with appropriate structural modification and other activity carried out by our research team.

\section{Acknowledgment}

We articulate our appreciation to authorities of V. P. \& R. P. T. P. Science College, Sardar Patel University, Vallabh Vidyanagar (Gujarat, India) for providing infrastructural \& chemical facilities. We are thankful to the Director, SICART, Vallabh Vidyanagar for spectral analysis facility. Authors are also grateful to the Microcare laboratory, Surat (Gujarat-India) for providing bioanalysis facility.

\section{References}

1. Singh N G, Nongrum R, Kathing C, Star Rani J W and Nongkhlaw R, Green Chem Lett Rev., 2014, 7(2), 137-144; DOI:10.1080/17518253.2014.902506

2. Akhaja T N and Raval J P, Med Chem Res., 2013, 22, 4700-4707; DOI:10.1007/s00044-013-0472-0

3. Akhaja T N and Raval J P, Eur J Med Chem., 2011, 46(11), 5573-5579; DOI:10.1016/j.ejmech.2011.09.023

4. Pratap U R, Jawale D V, Netankar P D and Mane R A, Tet Let., 2011, 52(44), 58175819; DOI:10.1016/j.tetlet.2011.08.135

5. Pratap U R, Jawale D V, Bhosle M R and Mane R A, Tet Let., 2011, 52(14), 16891691; DOI:10.1016/j.tetlet.2011.01.143

6. Billman J H and Diesing A C, J Org. Chem., 1957, 22(9), 1068-1070; DOI:10.1021/jo01360a019

7. Feng L S, Liu M L, Wang B, Chai Y, Hao X Q, Meng S and Guo H Y, Eur J Med Chem., 2010, 45(8), 3407-3412; DOI:10.1016/j.ejmech.2010.04.027

8. Gürsoy A, Ates Ö, Karali N, Cesur N and Kiraz M, Eur J Med Chem., 1996, 31, 643646; DOI:10.1016/0223-5234(96)89561-4

9. Rattan A, Antimicrobials in Laboratory Medicine, Churchill B I, Livingstone, New Delhi, 2000, pp.85-108.

10. Sahu R and Shrivastava S P, Chem Sci Trans., 2016, 5(2), 305-310; DOI:10.7598/cst2016.1190

11. Kateb B A, Hussien A K A, Kulkarni P A and Baseer M A, Chem Sci Trans., 2016, 5(2), 353-360; DOI:10.7598/cst2016.1215

12. Karaarslan M, Koparir P, Cansiz A, Orek C and Sap O, Chem Sci Trans., 2012, 1(1), 226-232; DOI:10.7598/cst2012.130

13. Kavitha R, Nagoor Meeran M and Sureshjeyakumar R P, Chem Sci Trans., 2015, 4(4), 1001-1006; DOI:10.7598/cst2015.1118 